\title{
TLR9 Agonist AST-008
}

National Cancer Institute

\section{Source}

National Cancer Institute. TLR9 Agonist AST-008. NCI Thesaurus. Code C157771.

A spherical nucleic acid (SN)-based agonist of toll-like receptor 9 (TLR9), with potential immunostimulating activity. Upon administration, TLR9 agonist AST-008 targets and is able to enter various immune cells, including monocytes/macrophages, plasmacytoid dendritic cells (pDCs), natural killer (NK) cells and B cells, through endocytosis. Within the endosome, it binds to and activates TLR9. TLR9 activation induces immune signaling pathways and activates various immune cells, including B-cells, pDCs, NKs, and induces both the production of T-helper 1 cells (Th1) and a Th1-mediated immune response as well as a cytotoxic T-lymphocyte (CTL)-based immune response against tumor cells. This results in an inhibition of tumor cell proliferation. TLR9 is a member of the TLR family, which plays a fundamental role in pathogen recognition and activation of innate and adaptive immune responses. The SNA is a dense, radial arrangement of nucleic acids (DNA) on the surface of liposomal nanoparticles, providing a 3D-construct, that has a high cellular uptake and an increased presentation of the DNA for TLR9 agonism. It also protects against breakdown by nucleases and increases the half-life of the construct compared to linear oligonucleotides that are not in SNA format. 